\title{
Free Volume in a PVME Polymer-Water Solution
}

\author{
Sara Capponi, Fernando Alvarez, and Dǔsan Račko*
}

Cite This: https://dx.doi.org/10.1021/acs.macromol.0c00472

Read Online

ABSTRACT: Molecular dynamics simulations of poly(vinyl methyl ether) in aqueous solution with weight concentration $c_{\mathrm{w}}=30 \%$ were performed in a wide temperature range by simulated cooling between 400 and $175 \mathrm{~K}$. A shift of glass transition temperature due to plasticization effect is observed with the decrease of simulated $T_{\mathrm{g}}^{\mathrm{MD}}$ by $25 \mathrm{~K}$ as compared to pure PVME at the same simulated cooling rate. The free volume is computed by using different probe radii $\left(R_{\mathrm{p}}\right)$. The free volume computed with $R_{\mathrm{p}}=0.5 \AA$ reproduces free volume amounts at the glass transition temperature from the free volume theories as well as thermal expansivity of the excess free volume given as $\alpha_{\mathrm{L}}-\alpha_{\mathrm{G}}$. A portion of $90 \%$ of the free volume is

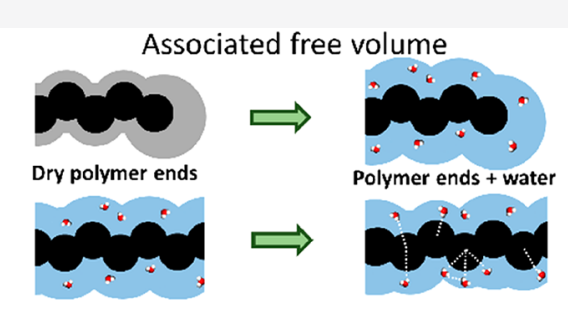

Hydrophobic groups + water formed by molecular bodies of both polymer and water molecules. In the presence of water molecules, the free volume of polymer ends shows only a miniscule increase by $7 \%$ as compared to the free volume around main-chain monomer units. The free volume around hydrophilic polymer groups involved in hydrogen-bonding formation is decreased, in agreement with the predicted relation between mobility and free volume. A picture hinting why percolation of the free volume is naturally connected with glass transition temperature is provided. By using the computed free volumes, we predicted a value of the orthopositronium (o-Ps) lifetime in the structure to be $\tau_{3}=1.31 \mathrm{~ns}\left(40.1 \pm 0.5 \AA^{3}\right)$ in cavities formed solely by monomer units, and $\tau_{3}=2.03$ ns for the average cavity in the structure.

\section{INTRODUCTION}

Poly(vinyl methyl ether) (PVME) belongs to the group of vinyl ether polymers whose synthetic production is well-known since 1928. However, the studies of the PVME itself could have started only after discovering a method of synthesis that allowed supplying of PVME in larger amounts. ${ }^{1-3}$ PVME is an amorphous polymer consisting of building blocks that contain hydrophilic and hydrophobic groups. It is an excellent system for studies of intermolecular interactions. An advantage of PVME is that it can be cross-linked by using $\gamma$-irradiation that removes the need for using additional cross-linker, making PVME an excellent candidate for possible biomedical applications. ${ }^{4-6}$ PVME is also miscible with other polymers forming blend nanocomposites.

PVME is hygroscopic and freely dissolves with water forming hydrogel solutions over a wide range of concentrations. ${ }^{8}$ PVME in water solutions is also used as a simplified model system for studying biological phenomena with relation to protein folding and water dynamics in a controlled environment defined more easily than in the case of aqueous protein solutions. ${ }^{9,10}$ In such solutions, water shows a complex behavior, particularly at concentrations lower than $c_{\mathrm{w}}=30 \%$, where water molecules are confined within the PVME matrix. ${ }^{11}$ As a result, water in low concentration does not form crystals but coexists with PVME forming a PVME-water complex because the water molecules are strongly bound to the polymer matrix. ${ }^{12-14}$ PVME in aqueous solutions gives rise to complex hydrogen-bonding networks with local hetero- geneities related to the presence of hydrophilic or hydrophobic groups. $^{15-17}$

Despite being an object of intense scientific investigations, PVME still poses challenges to experimental methods. For example, while PVME-water solution provides an excellent system for studying how water dynamics is affected by presence of a polymer, the dielectric spectroscopy (BDS) cannot reveal dynamics of the polymer itself, as the signal is hidden behind the one from water molecules. ${ }^{11}$ Similarly, in methods for studying dynamics of polymer chain such as quasielastic neutron scattering (QENS), speeding up of segmental dynamics in the presence of water pushes the fastest vibrational modes beyond the resolution of the experimental method. ${ }^{9}$ The high absorbance of the water molecules hinders IR spectroscopy, too. ${ }^{18}$

A highly scientifically interesting property, relating different structural, dynamic, and thermodynamic features by a single parameter, is the free volume. The free volume theory is one of the most successful concepts in polymer physics. However, its use for relating various properties is often disputed, arguing that the free volume is rather an abstract quantity tethered to

Received: March 2, 2020

Revised: $\quad$ May 8, 2020 
the particular definition how it was obtained. ${ }^{19-23}$ Recent progress in the field of the free volume theory has been done by White and Lipson, and their locally correlated lattice model (LCL), ${ }^{23}$ which is based on first-principles, does not inherently have connection to $T_{\mathrm{g}}$ or dynamics and quantifies free volume via thermodynamic/PVT data presuming a van der Waals component contained in the experimental volume. The LCL model provides an excellent prediction of glass transition temperatures and allows placing the different definitions of free volume within a physical picture of what the proposed contributions represent. Along with the progress in the field of theory, also two methods for direct determination of the free volume in polymers have been emerging.

One is the experimental method called positronium annihilation lifetime spectroscopy (PALS). From the number of experimental techniques employed to examine free volume, such as X-ray spectroscopy, neutron scattering, photochromic labeling, scanning tunneling microscopy, and transmission electron microscopy, the PALS is becoming arguably the most successful one for the direct examination of the local free volume holes in polymers. ${ }^{24}$ The PALS determines the free volumes in the structure based on annihilation behavior of positrons that relate the positronium lifetime to electron density by an empirical lifetime-to-volume correlation based on the quantum mechanical model of Tao, ${ }^{25}$ modified by Eldrup et al. $^{26}$ and by Nakanishi et al. ${ }^{27}$ for the temperature dependence of discrete lifetimes. In this correlation, the shape of the free volume hole is approximated to a sphere because an exact solution of electron-positron density functions in the highly irregular space is an unmanageable quantum mechanical problem. To account for the irregularity of the free volume cavities, other models also approximating the cavity shape to more complex geometries were developed, such as ellipsoidal, ${ }^{28}$ cubic, $^{29}$ or cylindrical. ${ }^{30}$ Recent theoretical and experimental studies suggest, though, that as the ratios of the main axes of symmetry remain preserved with temperature, the models would provide free volume amounts that would differ just by a constant value as a systematic bias in the investigated temperature range. ${ }^{31,32}$ It has to be noted that while the PALS is a successful technique in directly determining local free volumes, it still requires employment of additional techniques and considerations from existing free volume theories to yield free volume fractions. ${ }^{33-36}$ In addition, in the case of the PALS, similarly to other experimental methods mentioned above, water molecules (and polar groups on polymer) will be a hindering source for the signal-here known as positronium formation inhibition. Inhibition of the positronium arises from the presence of strongly electronegative groups of water molecules that would draw positively charged positrons and bias the PALS measurements. ${ }^{37,38}$

The second method for the direct determination of the free volume stems from molecular dynamics simulations by using atomistic models of molecules, with subsequent geometrical analyses of the intermolecular free volumes. The geometrical analyses can employ analytical methods by tessellation of space $^{39,40}$ or numerical integrations involving voxelized space. ${ }^{41}$ In Connolly's method, a boundary between molecular structure and its environment is calculated numerically by rolling a probe over a molecule and the volume of molecule within the generated surface is integrated, while the free volume is given as a complementary part to the computed molecular volume. ${ }^{42}$ When the size of voxels is decreased, the results would approach the volumes from the calculation of Connolly's solvent accessible surfaces. The volume integrations are computationally very expensive; hence, hybrid methods employing both voxel integration and analytical tessellations are being developed. ${ }^{43,44}$

Intrinsic limitation of the molecular dynamics simulations of condensed systems is different from those arising from the presence of water. Here, the intrinsic limitation relates to simulated cooling and the fact that with the current computational power of computers only very high cooling rates can be achieved, reaching up to 10 orders of magnitude faster cooling rate than in experiment, causing shift of the simulated glass transition temperature to higher values. Nonetheless, the molecular simulations can provide a useful picture on the structure and dynamics when bearing in mind scaling laws between the glass transition and the cooling rate.

In the present work, we investigate the free volume microstructure and its behavior with temperature in PVMEwater solution containing $c_{\mathrm{w}}=30 \%$ of water. Within the work we developed new methods allowing to analyze free volume amounts around specific structural constituents and allowing to make a prediction for measurable PALS free volume. As the free volume is considered being a measure of mobility, we analyze also the free volume around sites involved in hydrogenbonding network formation.

\section{METHODS}

Molecular Simulations. The molecules were built from scratch by initializing coordinates of all atoms of polymer and water. Then the structures were cleaned when the bond lengths and angles were adjusted to their equilibrium values according to hybridization. Next, the forces acting between atoms were treated by attributing condensed-phase optimized molecular potentials for atomistic simulations studies (COMPASS) molecular force field. ${ }^{45-48}$

Using these configurations of the polymeric chain and water, we built an amorphous cell by means of the amorphous cell module. The module implements a protocol based on an extension of well-established methods for generating bulk disordered systems, containing chain molecules in realistic equilibrium conformations. ${ }^{49}$ The prepared amorphous cell contained three polymeric chains and 414 water molecules. Each of the chains consisted of 100 monomers, giving 4248 atoms in the cell in total.

After the initial amorphous cell was generated, the molecular dynamics simulations were performed by using the DISCOVER module. At first, the potential energy was minimized, followed by a short molecular dynamics run. The structure was equilibrated under isobaric-isothermal thermodynamic conditions (number of particles, pressure, and temperature kept constant within NPT ensemble, using the isotropic stress tensor constraint) at $400 \mathrm{~K}$ until the conformational distribution; the size of the box and the potential energy fluctuated around constant values. A high temperature (400 K) was used to speed up equilibration and ease overcoming energetic barriers after generating the initial structure, as described by the high-temperature molecular dynamics protocol (HTMD). ${ }^{45}$ The HTMD often uses even higher temperatures. In our case, we employed only mediocre increase of the temperature, where our system was observed to be stable at least on the experimentally relevant time scales.

In the next step, the structure was dynamically equilibrated in the canonical ensemble (constant number of particles, 
volume, and temperature). The last structure from this NVT step was used as a starting point for data collection, recording structures every $0.01 \mathrm{ps}$ during molecular dynamics (MD) run of $1 \mathrm{~ns}$. The velocity-Verlet algorithm with a time step of $1 \mathrm{fs}$ was used for the integration of the equations of motion. ${ }^{50}$

To control the temperature, instead of a real temperaturebath coupling (Nosé-Hoover or Berendsen thermostat) a velocity scaling procedure with a wide temperature window of $10 \mathrm{~K}$ was applied. ${ }^{51}$

The structures at lower temperatures were obtained in an analogous way. Starting from the equilibrated structure at 400 $\mathrm{K}$, the temperature was lowered by $25 \mathrm{~K}$. After the first run, successive runs of $20 \mathrm{~ns}$, collecting data every $0.5 \mathrm{ps}$, were carried out. Within this procedure, we have obtained trajectories from production runs in a temperature range between 400 and $175 \mathrm{~K}$. From each of the trajectories we extracted 1000 uncorrelated structures for analyses of the free volumes for temperature well below the glass transition temperature $T_{\mathrm{g}}^{\mathrm{MD}}$, at $175 \mathrm{~K}$, at the $T_{\mathrm{g}}^{\mathrm{MD}}=250 \mathrm{~K}$, and well above $T_{\mathrm{g}}^{\mathrm{MD}}, 300 \mathrm{~K}$.

Free Volume Simulations. The free volume calculations were performed in voxelized space. The volume of the simulation box was sampled by a probe of given radius from the range $0 \leq R_{\mathrm{p}} \leq 2.5 \AA$. If no overlaps between inserted probe and any of the atoms of the molecules were found, the position of the probe was saved. The coordinates of saved probes indicated locations of the free probe accessible space, while also the numbers of atoms adjacent to the inserted probe were saved. The ID numbers of the atoms were later used to relate computed free volumes to their molecular environment and investigate the effects of hydrogen bonding or of particular constituents, like polymer ends or water molecules, on the free volume amounts. The volume of the inserted probes was integrated by using a very fine mesh of $0.1 \AA$.

In the next step the free volume cavities were constructed by finding contacts between the inserted probes. A cavity was defined as a set of probes for which the minimum distance between the probes forming a cavity was smaller or equal as a value given as $2 \times R_{\mathrm{p}}$. Further details on the computational method were provided in our earlier works. ${ }^{31,52}$ Because of extensive character of the free volume analyses, the results are collected for three temperatures: 300,250 , and $175 \mathrm{~K}$.

\section{RESULTS AND DISCUSSION}

Specific Volume and Glass Transition Temperature. In the studies of the free volume microstructure, usually the first data of interest to be compared with the experimental counterparts come with the simulated density or the specific volume. The density of the simulated bulk at given temperatures must reproduce the experimental values as well as possible since all additional volume in the simulated structures would transfer directly to the simulated free volume amounts. Figure 1 shows the specific volumes (reciprocal density) obtained by simulated cooling for pure PVME and the mixture of PVME with water $\left(c_{\mathrm{w}}=30 \%\right)$. The specific volume of the pure PVME was obtained in the previous simulation work and showed an excellent agreement with experimental values in the region between the simulated glass transition temperature and $300 \mathrm{~K}^{31}$ The simulated glass transition temperature was found to be $275 \mathrm{~K}$, shifted toward higher values with respect to the experimental value $(250 \mathrm{~K})$ due to the effects of the cooling rate. The shift of glass transition is typically encountered in the molecular dynamics simulations as

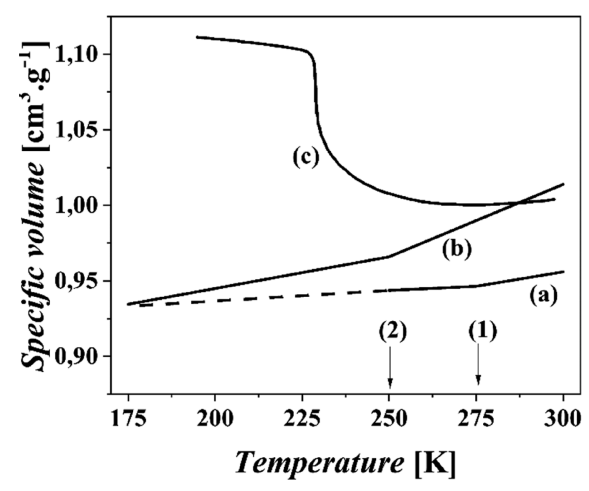

Figure 1. Specific volume as a function of temperature for (a) MD simulations of pure PVME; ${ }^{31}$ (b) MD simulations of aqueous solution of PVME with concentration of water, $c_{\mathrm{w}}=30 \%$, and (c) predicted for pure water. $^{63}$ The arrow indicates simulated glass transition temperature for pure PVME (1) and PVME in solution (2). The dashed line indicates extrapolated specific volumes in the glassy region toward $175 \mathrm{~K}$, obtained for previous simulations of pure PVME.

result of a dynamic arrest, as the experimental values of the cooling rate cannot be obtained in practically feasible computational times. The shift of the glass transition temperature with the cooling rate can be calculated by using Donth's formula relating the shift of the glass transition temperature and cooling rate by fragility of the system by a Vogel-Fulcher type of equation, $T_{\mathrm{g}}^{\mathrm{MD}}=T_{\mathrm{g}}\left(1-\Delta \log \phi_{\mathrm{c}} /\right.$ $\left.m_{\rho}\right)^{-1.53}$ The cooling rate in the simulations was $10 \mathrm{~K} / 1 \mathrm{~ns}$, giving the difference $\Delta \log \phi_{\mathrm{c}}=10$ orders of magnitude from the experimental MTDSC cooling rate $1 \mathrm{~K} / \mathrm{s}{ }^{11}$ The glass transition temperature at the achieved cooling rate in the simulations $T_{\mathrm{g}}^{\mathrm{MD}}=275 \mathrm{~K}$ is obtained from Donth's formula for the fragility of pure PVME $m_{\rho}=75,{ }^{54}$ and value of $T_{\mathrm{g}}=240 \mathrm{~K}$, which well falls into interval of experimentally measured values $T_{\mathrm{g}}=247 \pm 15 \mathrm{~K}^{11,55}$ As a justification of our approach to estimate the shift of $T_{\mathrm{g}}$ with the cooling rate, we would like to stress that despite the shift of the glass transition with the cooling rate being a well-known phenomenon, the difference between cooling rates encountered in laboratory experiments would not usually exceed 3 orders of magnitude. In this case, the dependence of $T_{\mathrm{g}}$ on $\phi_{\mathrm{c}}$ is also often approximated by a linear extrapolation. To justify the validity of such an extrapolation approach over much larger orders of magnitude of $\phi_{c}$, such as encountered in our molecular simulations, Baschnagel et al. examined the dependence of the $T_{\mathrm{g}}$ over 3 orders of magnitude at very high cooling rates by means of computer simulations. ${ }^{56}$ The authors suggested that on wider ranges of cooling rates the behavior should be approximated rather by a Vogel-Fulcher type of behavior, as the cooling rate affects the system differently on different length scales. In a recent experimental work, the effect of cooling rate was studied over 6 orders of magnitude by a revolutionary flash DSC technique. ${ }^{57}$ The fits over the measured $T_{\mathrm{g}}$ 's vs $\phi_{\mathrm{c}}$ showed that the Vogel-Fulcher type of equation provides the best match for the observed behavior.

Figure 1 also shows the temperature dependence of the specific volume of the PVME-water mixture and the specific volume of pure water. The calculated specific volume of the simulated PVME + water structure $\left(c_{\mathrm{w}}=30 \%\right)$ cannot be compared to the experimental values directly due to lack of such data. In existing works, the specific volumes of PVMEwater mixtures were found to be above the specific volume of 

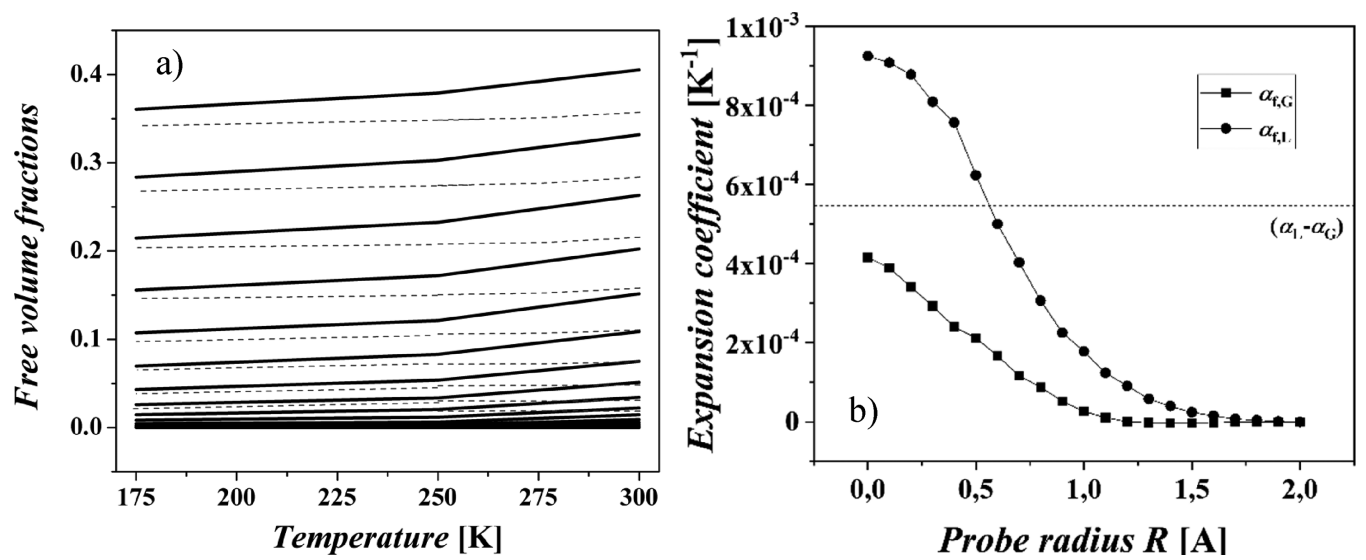

Figure 2. Computed free volume fractions. (left) Computed free volume fractions as a function of temperature in aqueous solution of PVME (solid lines) and pure PVME (dashed lines). The fractions are computed for probes with different radii, from top to bottom 0 (dot probe), $0.1,0.2$, etc., up to $1.2 \AA \AA$. (right) Coefficient of thermal expansion of the excess free volume computed as a function of probe radius in glass phase and liquid phase. The dashed line shows the value of the $\left(\alpha_{\mathrm{L}}-\alpha_{\mathrm{G}}\right)$ term from Simha-Boyer theory.

water in experimental studies with a water content of $c_{\mathrm{w}}=$ $80 \%{ }^{55}$ and by simulations with a water content $c_{\mathrm{w}}=50-$ $75 \% .^{16,17}$ In our simulations, the specific volume of the PVME-water mixture with a water content of $c_{\mathrm{w}}=30 \%$ is also found between the values of the pure PVME and pure water in the greater part of the investigated temperature region. At high temperature above $290 \mathrm{~K}$ the specific volume of the PVMEwater mixture raises above the specific volume of water. This could be attributed to destroying the hydrogen-bonding network between water and polymer molecules with rising temperature as suggested in earlier work. ${ }^{17}$ Note that the PVME-water mixture is known to exhibit lower critical solution temperature (LCST) and demixing of phases at higher temperature. With the current concentration of water $c_{\mathrm{w}}=$ $30 \%$, the experimentally observed LCST occurs at $310 \mathrm{~K},{ }^{58}$ and hence we cannot relate this phenomenon to the observed increase of the specific volume. Moreover, the glass transition temperature obtained in the simulations of the PVME with water $T_{\mathrm{g}}=250 \mathrm{~K}$ is lower than the glass transition temperature obtained for the pure PVME at the same cooling rate. The decrease of the glass transition temperature can be attributed to the plasticizing effect of water. ${ }^{59,60}$ The glass transition temperature obtained in simulation is again higher than that experimentally measured in the PVME with the given water content $30 \%, T_{\mathrm{g}}=210 \pm 15 \mathrm{~K} .{ }^{11,55}$ The observed shift of the glass transition temperature in the simulations with the difference of cooling rate $\Delta \log \phi_{c}=10$ orders of magnitude, and the considered experimental glass transition temperatures $200-210 \mathrm{~K}$ would give the corresponding fragility parameter $m_{\rho}=45-62$ for the polymer-water mixture. The obtained fragility is lower than the value for the pure PVME, which is consistent with the expectation that water content should decrease the fragility. ${ }^{61}$ The shift of the glass transition with cooling rate in the wet PVME is larger $\Delta T_{\mathrm{g}}=40 \mathrm{~K}$ than that observed in the case of pure PVME, $\Delta T_{\mathrm{g}}=25 \mathrm{~K}$. This is due to different fragilities and different glass transition temperatures entering the Donth formula. A similar behavior, i.e., increase of the $\Delta T_{\mathrm{g}}$, with the increasing concentration of plasticizer was reported by Zhou et al. in recent computer simulation study of plasticized poly(vinyl chloride). ${ }^{62}$ The authors also explored effects of cooling rate on shorter scales around molecules of plasticizer and employed Vogel-Fulcher-Tammann equation for extrapolations to support their conclusions about shifts of the $T_{\mathrm{g}}$ with the cooling rate.

Free Volume Fractions. The fractional free volume represents the free volume related to the total volume of the sample. In computer simulations, it is calculated from computed amounts of the free volume by dividing them with the volume of the simulation box. Despite the free volume theory being one of the most successful concepts in polymer physics, it is often argued that using the free volume for explaining behavior of various properties is controversial, as the free volume cannot be measured directly, and its meaning is tethered to the particular free volume theory and the way it is obtained. As we described in the Introduction, the recent development in the field of the free volume theory provided a derivation of the new predictive model for the free volume based on the first-principles ${ }^{23}$ and also two emerging methods for direct determination of the free volume allowed studying yet unprecedented aspects of the free volume. In our current work as well as in our previous simulations, ${ }^{31}$ we show that various free volume measures can be computed directly in a very straightforward way. In the following paragraphs, we put the calculated free volumes in context with the existing free volume theories, while the aim of the discussion will be to show that our computational model provides reasonable amounts of the free volume. Later, the effect of water molecules on the obtained distributions of the free volume around specific sites will be discussed.

Figure $2 \mathrm{a}$ shows the free volume fractions as a function of temperature computed for various probe radii. The free volume obtained by probing of structure corresponds to a volume enclosed by Connolly's solvent accessible surface area. If a point probe is used, i.e., a probe with a zero radius, the free volume obtained is defined as the empty space volume. The free volume is a complementary part of the space that is occupied by atoms and hence is often called the occupied volume. ${ }^{64}$ A complementary part to the empty space calculated with a point probe is the van der Waals volume of molecules. In the existing free volume definitions this van der Waals volume of molecules is considered to be constant with temperature and represents the volume excluded by hardcore spheres. From the calculations employing a very fine mesh we do see a slight dependence of the van der Waals volume on temperature, while the molecular volume at $300 \mathrm{~K}$ is increased 
by about $1.2 \%$ against its original value at $175 \mathrm{~K}$. This slight variation probably originates from the fact that the molecular potential employed is not a hard-core potential. The smallest portion of the empty space fraction $36.5 \%$ was computed at $175 \mathrm{~K}$. This value is close to the empty space in the cubic close packing of hard-core spheres given by the value $36 \% .{ }^{65}$ Despite a similar observation being made for the pure PVME for temperatures well below $T_{g}$, the fact that the empty space fraction approximates the fraction of the empty space in the dense packing of hard-core sphere may be coincidental, as van der Waals spheres surrounding atoms in our atomistic simulations are not mutually repulsive hard-core spheres and do overlap. Hence, the appropriate limiting fraction of the empty space should be evaluated by appropriate models such as the concentric shell model (see next).

Models beyond Doolittle's equation ${ }^{66}$ introduced a picture of expanding occupied volume where the occupied volume expands due to vibrations of atoms. Here, the volume of molecules with the contribution of the expansion is still called occupied volume while the complementary free space is called excess free volume, as thoroughly reviewed by Lipson and White. ${ }^{23}$ In the most straightforward approach, we approximate the contribution of the vibrational expansion by increasing the volume of the probe employed to calculate the "excess" free volume amounts. In the following, we examine in light of the different free volume theories how employing probes with different radii for the free volume calculation agrees with predictions of the behavior of the free volume. First of all, one of the earliest assumptions in the classical free volume theories such as Williams-LandelFerry $^{67}$ or Simha-Boyer ${ }^{68}$ was the existence of a universal value of the free volume at $T_{\mathrm{g}}$. Such a "universal" value of the free volume employed in these different theories differs from $2.5 \%$ to $11.3 \%$ for the excess free volume, and it has been obtained as average from different values determined for different polymers. For example, the values of the excess free volume at $T_{\mathrm{g}}$ in the data set used by Simha and Boyer varied between $8.1 \%$ and $14 \%{ }^{68}$ It has to be noted that the existence of such a universal value and of the iso-free-volume state was disproved by the recent LCL model, ${ }^{23}$ and it cannot be confirmed by our molecular dynamics studies. ${ }^{31,69}$ In Figure $2 \mathrm{a}$, we show the fractional free volume computed as a function of temperature and radii of the probes employed. Employment of different probe radii to approximate vibrational expansion of atoms also provides a free parameter in the computational model, allowing us to interpret the computed free volume amounts with predictions from different free volume theories. Figure 2 infers that the amount of the free volume at $T_{\mathrm{g}}$ dramatically drops as the probe radius increases. As discussed above, the value obtained for a point probe with zero radius is about $36 \%$, which is still larger than the value predicted by Simha-Boyer theory for the total free volume $\left(\alpha_{\mathrm{L}}\right) T_{\mathrm{g}}=(9.95$ $\left.\times 10^{-4} \mathrm{~K}^{-1}\right) \times 250 \mathrm{~K}=24.9 \%$, where we used the coefficient of cubical expansion $\alpha_{\mathrm{L}}$ obtained from the simulated cooling above the simulated $T_{\mathrm{g}}^{\mathrm{MD}}$. It is noteworthy that the expansion coefficient $\alpha_{\mathrm{L}}=9.95 \times 10^{-4} \mathrm{~K}^{-1}$ in wet PVME is larger than that obtained from simulations of pure PVME, $\alpha_{\mathrm{L}}=6.78 \times$ $10^{-4} \mathrm{~K}^{-1}$. In the case of pure PVME, the expansion coefficient obtained by employing our simulation protocol provided excellent agreement of the computed value with the experimental data, with a deviation of only $8 \times 10^{-6} \mathrm{~K}^{-1}$ $(1.2 \%){ }^{31}$ In the case of the wet PVME, we were unable to conduct such a precise comparison due to lack of the data in the literature; however, the computed values qualitatively follow the prediction by the Simha-Boyer rule for the expansion coefficient $\alpha_{\mathrm{L}} \sim 0.113 / T_{\mathrm{g}}$.

Furthermore, Simha and Boyer predicted that the amount of the excess free volume at $T_{\mathrm{g}}$ should have a universal value that corresponds to $\left(\alpha_{\mathrm{L}}-\alpha_{\mathrm{G}}\right) T_{\mathrm{g}}=11.3 \%$. By using their relation and plugging in the coefficients of cubical expansion and $T_{\mathrm{g}}$ from our simulations, we obtain the value $\left(\alpha_{\mathrm{L}}-\alpha_{\mathrm{G}}\right) T_{\mathrm{g}}=(9.95$ $\left.\times 10^{-4} \mathrm{~K}^{-1}-4.47 \times 10^{-4} \mathrm{~K}^{-1}\right) \times 250 \mathrm{~K}=13.4 \%$. As shown in Figure $2 \mathrm{a}$, the free volume fractions corresponding to $11-13 \%$ are determined by the probe with radius $R_{\mathrm{p}}=0.4-0.5 \AA$. Next, the slope of the excess free volume in the region above $T_{\mathrm{g}}$ given as $\alpha_{\mathrm{L}}-\alpha_{\mathrm{G}}$ would correspond to the coefficient of thermal expansion of the excess free volume $\alpha_{\mathrm{L}}^{f}$ determined from the free volume, calculated with the probe radii between 0.5 and $0.6 \AA$ (Figure $2 \mathrm{~b}$ ). The assumption that the excess free volume in glassy region would follow the slope of the thermal expansion, $\alpha_{\mathrm{G}}$, for any probe other than that with zero radius is hard to justify and would ignore experimental curvatures, as already pointed out by Simha and Boyer. ${ }^{68,70}$ Similarly, in the case of pure PVME the free volume fraction at $T_{\mathrm{g}}$ computed by the predictive relation derived by the Simha-Boyer theory would be $\left(\alpha_{\mathrm{L}}-\alpha_{\mathrm{G}}\right) \mathrm{T}_{\mathrm{g}}^{\mathrm{MD}}=\left(6.78 \times 10^{-4} \mathrm{~K}^{-1}-3.00 \times 10^{-4}\right.$ $\left.\mathrm{K}^{-1}\right) \times 275 \mathrm{~K}=10.4 \%$. This is again close to the universal value $11.3 \%$, and it corresponds to the value obtained by the probe with radius $R_{\mathrm{p}}=0.5 \AA \AA^{31}$

Despite the good agreement of our computed free volume data with the Simha-Boyer theory, one has to be aware that the free volume amounts predicted by other models could be different. The LCL model by White and Lipson ${ }^{23,71,72}$ yields free volume fraction for the pure PVME at the simulated $T_{\mathrm{g}}^{\mathrm{MD}}$ $=275 \mathrm{~K}$ to be $f=1-\left(r / M_{\mathrm{w}}\right) \nu \rho=1-111.53 \mathrm{~mol} \mathrm{~kg}^{-1} \times$ $7.9296 \times 10^{-6} \mathrm{~m}^{3} \mathrm{~mol}^{-1} \times 1 / 0.9536 \times 1000 \mathrm{~kg} \mathrm{~m}^{-3}=7.3 \%$, i.e., lower than that computed by the molecular simulations for the probe with $R_{\mathrm{p}}=0.5 \AA$. The value predicted by the LCL would be obtained for the probe with the radius $R_{\mathrm{p}}=0.6 \AA \mathrm{.}^{31}$ The LCL model allows straightforward derivation of the predictive relation also for the mixtures and blends. ${ }^{73-75}$ However, the molecular parameters $r$ and $\nu$ for water are currently missing. Because of the complex PVT behavior of water, obtaining these parameters by using the fitting protocol available in the literature may represent a challenge. Nonetheless, we believe that the aforementioned discussion of the computed free volumes accumulated enough theoretical support that our computational model provides physically reasonable amounts of the free volume and these can be used in further discussion of the local free volumes and the effect of water molecules on distributions of the free volume around specific sites.

Additionally, to justify employment of the expanded (nonzero) probe radius to capture expansion of the occupied volume due to vibrations, we analyzed free volume in 50000 structures of pure PVME in a molecular dynamics trajectory that were separated by $20 \mathrm{fs}$ and overlapped. ${ }^{76}$ The picture we obtained showed that the free volume structure obtained is dependent on the time window employed to overlap the structures. When the free volume was calculated on the structure overlapped over 1 ps, hence in the vibrational domain, the free volume obtained was very similar in glassy and liquid states. The free volume that existed below $50 \%$ of the time defined by the time window represented a shell around atoms about $0.5 \AA$ thick. 
The probe radius around $0.5 \AA$ is interesting, as it corresponds to the radius of the o-Ps atom. The probe of radius $0.53 \AA$ was used by Arizzi et al. to predict the size of the free volume cavities measured by PALS, and it is used in our models to simulate the PALS free hole volume. ${ }^{31,77}$ The probe with the radius of $0.53 \AA$ is large enough to break the continuous free volume space computed for a point probe into separate cavities. However, the probe is also smaller than any of atomic radii and hence it is still able to probe interatomic spaces. As a result, employing the probe with radius $0.53 \AA$ produces bimodal distributions of the free volume, where the first peak corresponds to atomic packing and the main peak corresponds to large free volume cavities formed by packing of polymer segments. ${ }^{31}$ The peak of the free volume distribution corresponding to the atomic packing is consistent with the picture drawn by Duda and Vrentas, who argued that a portion of empty space is not available for movements of molecules and transport processes as it is associated with a large redistribution energy, and they called this portion interstitial volume. ${ }^{78}$ In the simulation of the PALS free hole volume the portion of the smallest cavities corresponding to the atomic packing are neglected, believing that these are not accessible to o-Ps localization, while positronium can see only the true holes. After exclusion of the interstitial free volume cavities, the free volume fraction drops to $\sim 3-6 \%$ at $T_{g},{ }^{31,69}$ that is, a value closer to the free volume amounts predicted by the WilliamsLandel-Ferry to be around $2.5-3 \%{ }^{67}$

Figure 2a also shows that the free volume fractions for increasing radii of probes steeply drop, and the structure cannot contain probes with radii larger than $2 \AA$. This also indicates that in the experiments employing even the smallest molecular probes, such as electron spin resonance (ESR) or fluorescence probes, ${ }^{79-81}$ these probes must create their own free volume. In the case of the PALS such a bias arising from the interference of molecular probes with polymer environment is not encountered. ${ }^{32}$

The free volume fractions obtained in the PVME + water structures are larger than those calculated for the pure PVME due to swelling of wet polymer, as expected also from the temperature dependence of the specific volume shown in Figure 1. Figure 3 shows the normalized free volume fractions as a function of probe radius. This representation is typically

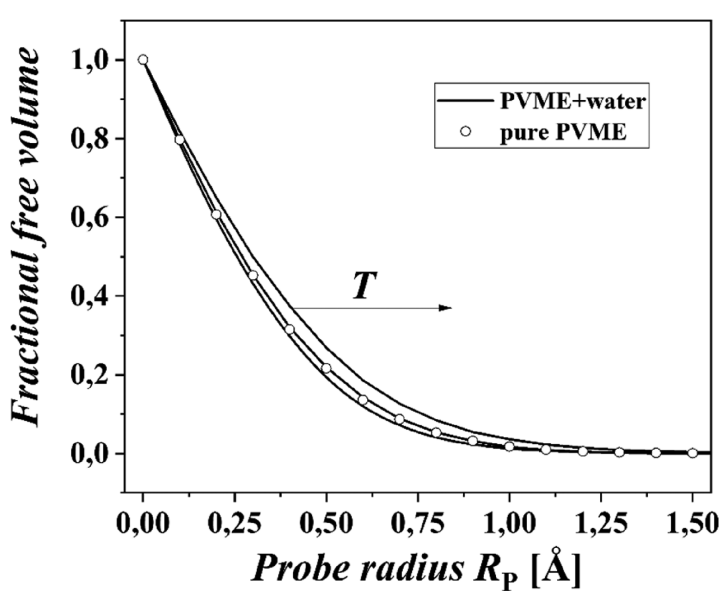

Figure 3. Porosity, i.e., the free volume fractions computed for three different temperatures, in the indicated direction the curves correspond to $175, T_{\mathrm{g}}^{\mathrm{MD}}=250$ and $300 \mathrm{~K}$. The empty circles correspond to the porosity in pure PVME. ${ }^{31}$ used to show porosity data. The free volume fractions computed in the PVME + water system for three different temperatures are shown. The dependence of the free volume fraction on the probe radii shows an exponential decay behavior which is characterized by a steep descent that declines into an exponential tail. In the region where the dependence declines to the exponential tail the maximum of cavity numbers occurs. $^{31}$ Figure 3 also shows that despite larger free volume amounts occurring in the PVME + water system than in pure PVME, the dependence of the free volume fraction follows approximately the same decay. This indicates that the structure of the free volume would consist of a larger amount of smaller free volume cavities. The origin of the smaller cavities in the PVME + water system is probably related to smaller effective radius of constituents in the packing introduced by water molecules.

The equation relating the free volume fraction in a set of partially overlapping spheres given as a function of sphere radii, $R_{\mathrm{e}}$, density, $\rho$, and hardness parameter, $\epsilon$, is given by the concentric shell model. ${ }^{82}$ The hardness is a model parameter expressing the extent of the mutual overlaps of the spheres in the packing with the value fixed between 0 for randomly overlapping spheres to 1 in packing of perfectly hard-core spheres. It has been shown that spheres in packing used to represent molecular structures of polymers tend to overlap, reaching values around $\epsilon=0.2-0.3{ }^{31,83}$

The equation of the concentric shell model was fitted over the calculated free volume dependences in Figure 3. The density and the number of spheres in the packing were fixed parameters, while the number of spheres corresponded to number of polymer segments $300+414$ water molecules, or 700 polymer segments in pure PVME. The effective radius of probes in the packing of segments of pure PVME was $R_{\mathrm{e}}=4.45$ $\AA$, and as expected, it is larger than the effective radius of structural constituents in PVME + water, $\left\langle R_{\mathrm{e}}\right\rangle=3.32 \AA$, for the investigated range of temperatures. The effective radius $R_{\mathrm{e}}$ in polymer water mixture varies from 3.42 below $T_{\mathrm{g}}^{\mathrm{MD}}$ to 3.23 at $300 \mathrm{~K}$, probably reflecting decreased clustering of water molecules at higher temperatures. The hardness parameter in pure PVME reached $\epsilon=0.3$ while in the PVME + water system $\epsilon=0.25$.

Free Volume Distributions. In the next step, cavity volumes are calculated. The free volume cavities are defined as a set of $N_{\mathrm{p}}$ adjacent probes $P_{i}\left(\mathbf{r}_{i}\right)$ inserted into the structure during determination of the free volume fractions, such that the lowest distance among the probes forming a cavity is smaller than a certain threshold value, given as a double of the employed probe radius, $\forall i, j \in N_{\mathrm{p}}, i \neq j: \min \left(\left|\mathbf{r}_{i}-\mathbf{r}_{j}\right|\right) \leq 2 R_{\mathrm{p}}$. The shape of the free volume cavities is highly irregular, and thus even the cavities with large volumes cannot be constructed just by using larger probes. ${ }^{31,84-86}$ Figure 4 shows the free volume distributions of the cavity volumes obtained in the simulated structure of PVME + water at $175 \mathrm{~K}$. The cavity volume distributions are calculated separately for polymer phase, water phase, and interfacial cavities that are formed by both polymer segments and water molecules. These contributions are normalized to unity as $V\left(V_{c}\right) / \max \left(V\left(V_{c}\right)\right)$. As shown in the case for the pure PVME, the distributions of cavity volume show a bimodal behavior. The first peak of the distribution in the region between 1 and $10 \AA^{3}$ belongs to cavities consisting of only a few probes $P_{i}$. These smallest cavities sample the interstitial space between atoms. The second peak occurs in the region between 10 and $100 \AA^{3}$ and 


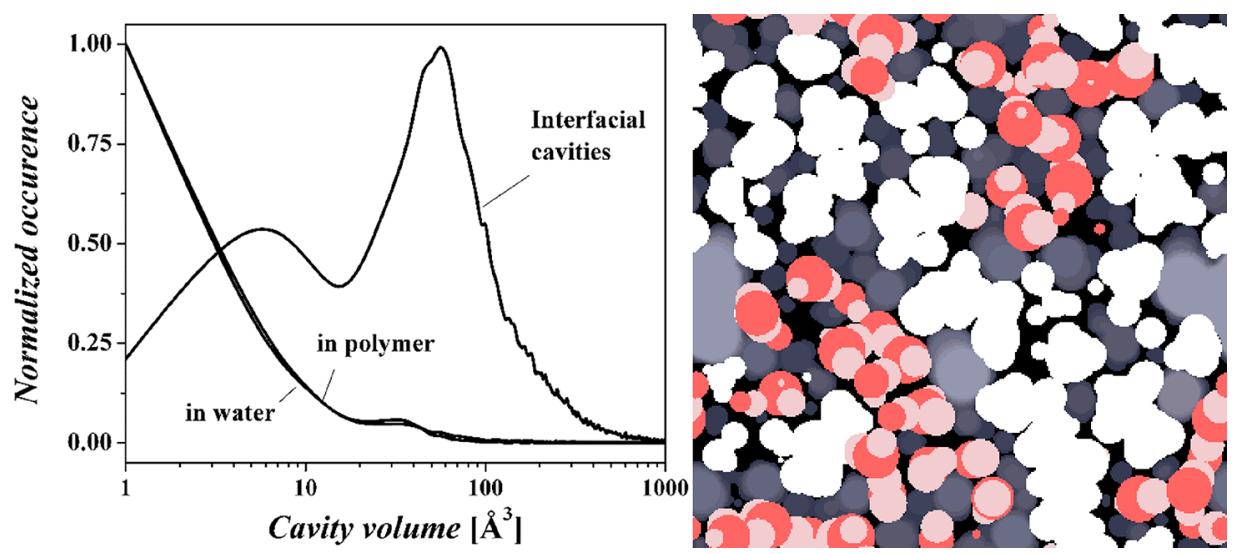

Figure 4. (left) Free volume distributions as a function of the volume of free volume cavities normalized to the maximum of the distribution. The corresponding distributions of the free volume cavities formed solely by water molecules, by polymer molecules, and by both water and polymer molecules are indicated. (right) A slice of the molecular structure with the free volume. White regions correspond to atoms belonging to PVME, reddish color shows the volume occupied by water molecules (darker oxygen, lighter hydrogen atoms), free volume regions are shown in gray scale, with darker colors being the interstitial volumes and lighter colors corresponding to larger volumes.

corresponds to true "hole" free volumes. Table 1 summarizes the contributions to the free volume in absolute numbers. The

\section{Table 1. Partitioning of Free Volume Cavities into Phases}

\begin{tabular}{lccc} 
& water phase & polymer phase & interphase \\
\hline cavity volume, \% & 1.7 & 8.4 & 89.9 \\
cavity number, \% & 14.0 & 27.6 & 58.4 \\
maximum cavity, $\AA^{3}$ & 68.8 & 119.1 & 854.6 \\
\hline
\end{tabular}

free volume in pure phases of water and polymer represents only $10 \%$ of the total free volume present in the structure, suggesting a high degree of mixing of the phases. It also shows that most of these cavities found in pure phases corresponds to interstitial volumes between atoms, although scarce cases of large cavities can be formed also in the separate phase reaching maximum volume of about $100 \AA^{3}$ with a slight peak forming at $30 \AA^{3}$. Larger amounts of the interstitial cavities around the body of the polymer are most likely related to the rugged topology of the polymer and its methoxyl side groups.

The volume of the largest cavities found to be formed exclusively by molecular bodies of a given phase is much smaller than the volume of the largest cavities found in the interfacial region, with volume approaching $1000 \AA^{3}$. The data also show that despite the contribution of the free volume in the pure water phase being only about $1.7 \%$, these cavities at the same time represent $14 \%$ of all cavities. Also, the height of the prepeak belonging to interstitial cavities with volume below $10 \AA^{3}$ in the mixed polymer water phase is larger, reaching $\sim 0.53 \%$ of the main peak, that in the pure PVME $\sim 30 \%$. $^{31}$ This is consistent with the behavior discussed with the data in Figure 3, suggesting that despite the larger free volume amounts in the PVME + water mixture than in pure PVME, the cavity sizes are about the same due to large numbers of smaller cavities created by the presence of water molecules.

In Figure 5, the distributions of the free volume around particular constituents of the molecular structure were computed in the structure at simulated glass transition $T_{\mathrm{g}}^{\mathrm{MD}}$ $=250 \mathrm{~K}$, while the occurrence of cavity volumes around polymer monomers, polymer ends, and water molecules is calculated. The cavity volumes were normalized to the volume of the constituents of the molecular structure or structural unit. The distributions show that the "excess" free volume around

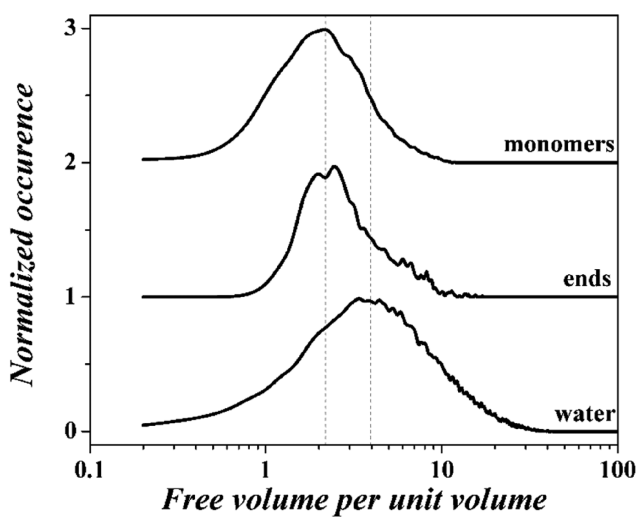

Figure 5. Normalized distribution of cavity volumes around water molecules, main chain monomers, and polymer ends. The volume of cavities is normalized by relating the cavity volume to the volume of the building unit: polymer end $69.5 \AA^{3}$, main-chain monomer $67.1 \AA^{3}$, and water molecule $19.5 \AA^{3}$.

water molecules is relatively higher than that around the polymer segments, with a peak at $8.22 \pm 0.01$ obtained by lognormal fit $\left(R^{2}=0.998\right)$. In addition, the free volume around main-chain monomers was calculated where only the free volume of cavities formed by monomers separated more than 10 monomers from the end units (two Kuhn segments) were considered. The result from this computation shown in Figure 5 is that the computed cavity volumes do exhibit a small difference of about $7 \%$ with peaks at $3.76 \pm 0.01\left(R^{2}=0.991\right)$ for polymer ends and $3.49 \pm 0.01\left(R^{2}=0.991\right)$ for main-chain monomers. This is much less than that observed in pure polymeric systems showing increase of the free volume by $55 \% .{ }^{87}$ Because of the lower number of bonds connecting the chain ends making them effectively "free", the chain ends are associated with higher amounts of free volume. ${ }^{88}$ In the FloryFox theory, ${ }^{64}$ the polymer ends act as impurities effecting thermodynamics properties and glass transition temperature as result of higher mobility of polymer if a larger number of polymer ends are present. On the other hand, the Fox equation for the glass transition temperature of a mixture describing the effect of plasticization predicts that the glass transition temperature should follow an inverse average $1 / T_{\mathrm{g}}=w_{1} / T_{\mathrm{g} 1}$ $+w_{2} / T_{\mathrm{g} 2} \cdot{ }^{89}$ Because the water molecules have a larger free 

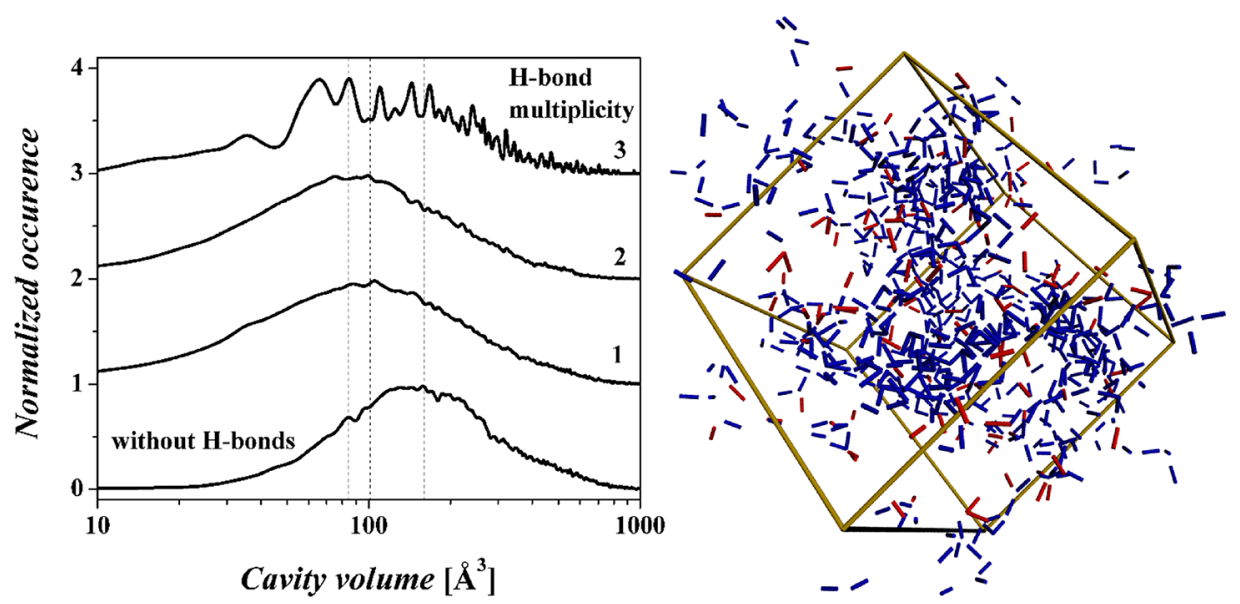

Figure 6. (left) Cavity volume around hydrophilic methoxyl side groups on PVME polymer as a function of the multiplicity of hydrogen bonds. Medians (average cavity volumes) obtained from the log-normal fits are $226.7 \pm 1.6 \AA^{3}\left(R^{2}=0.992\right)$ for side groups without $\mathrm{H}$-bonds, $202.2 \pm 1.8$ $\AA^{3}\left(R^{2}=0.996\right)$ for single H-bonds, $170.4 \pm 1.4 \AA^{3}\left(R^{2}=0.995\right)$ for bifurcated H-bonds, and $180.5 \pm 8.8 \AA^{3}\left(R^{2}=0.866\right)$ for trifurcated H-bonds. The dashed lines indicate the position of the peak obtained from log-normal fits. (right) Snapshot of hydrogen-bonding network; blue sticks show hydrogen bonds between water molecules, and red sticks show hydrogen bonds between water and hydrophilic groups of the polymer.
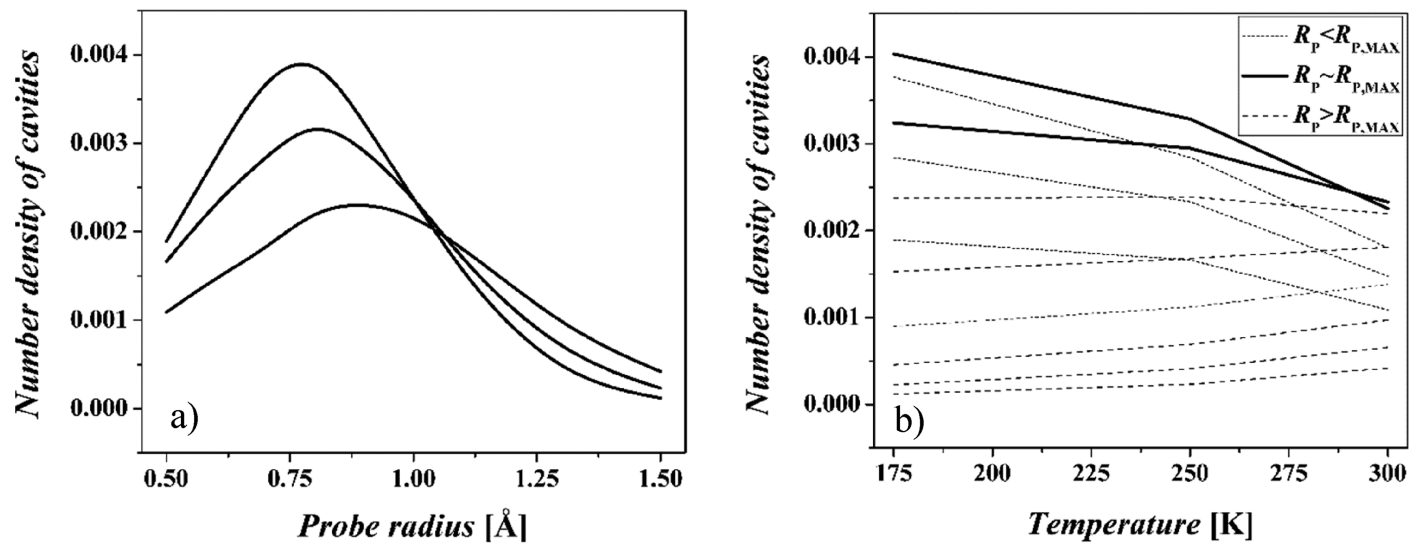

Figure 7. Distributions of the number of free volume cavities. (left) Distributions of the total number of free volume cavities as a function of probe radii. (right) Dependence of the total number of the free volume cavities as a function of the temperature for a given probe radius. The dotted lines correspond from bottom to the top to radii 0.5, 0.6, and $0.7 \AA$; solid lines represent from top down 0.8 and $0.9 \AA$ (peak maximum); dashed lines from top to bottom correspond to probe radii 1.0, 1.1, 1.2, 1.3, 1.4, and $1.5 \AA$.

volume associated with them, which is connected also with the lower $T_{\mathrm{g}}$ of water and $\sim 100$ times larger amount of water molecules as compared to polymer ends, the water molecules can be taking control over chain dynamics. Capponi et al. studied dynamics in aqueous PVME solution with concentrations of $c_{\mathrm{w}}=30 \mathrm{wt} \%$ of water in the polymer. ${ }^{9}$ In a combined QENS/DSC experimental work, they observed a shift of $T_{\mathrm{g}}$ by $37 \mathrm{~K}$ as compared to pure PVME due to plasticization effects, and the dynamics of wet PVME was 5 times faster, while they were not able to further follow the dependence of the characteristic dynamic times toward the $T_{\mathrm{g}}$, due to limited resolution of NS. Consistent with the picture of chain dynamics controlled by water, Pastorczak et al. suggested in their combined Raman spectroscopy, dielectric spectroscopy, and calorimetry experimental study that water in the presence of PVME polymer starts premelting already at $213 \mathrm{~K}$, which triggers also the first segmental motions of polymer. ${ }^{90}$

As shown in experimental and computational works, PVME in water solution gives rise to a complex hydrogen-bonding network formation. Tamai et al. studied the hydrogen-bonding network in PVME by means of molecular dynamics simulations. ${ }^{16,17}$ They observed that there exist regions with increased and decreased ability for hydrogen-bonding formation around the polymer based on the hydrophilic or hydrophobic part of the polymer exposed to water molecules. They further observed that the ability of hydrogen-bonding formation at a given region has a long-range effect on the surrounding water molecules, while regions with a pronounced or decreased hydrogen-bonding network were observed. Capponi et al. used this picture to interpret dynamic heterogeneities around the wet polymer. As dynamics and mobility are eminently related to the free volume amounts in the free volume theory, we calculated the distributions of the free volume around the polymer as a function of multiplicity of hydrogen bonds between water and the methoxyl group of the polymer, shown in Figure 6.

First, the hydrogen bonds were analyzed by considering as a hydrogen bond any contact between water hydrogen and methoxyl oxygen atoms closer than $2.6 \AA$ and the angle between acceptor (oxygen in the methoxyl group), proton (hydrogen of a water molecule), and donor (oxygen in the water molecule) larger than $120^{\circ}$. The highest multiplicity of the hydrogen bonds found in the structure was 3 . The occurrence of trifurcated hydrogen bonds is statistically rare, 
which is responsible for the structured (noisy) looks of the corresponding distribution. The positions of peaks in Figure 6 show that if the methoxyl group is involved in hydrogenbonding formation with higher multiplicity, the cavity free volume associated with it and hence the mobility are decreased. The largest free volume cavities were found around groups that were not involved in hydrogen-bonding formation. This observation obtained from the current simulations is also consistent with the free volume measurements by PALS in systems creating hydrogen-bonding networks. ${ }^{91,92}$

Cavity Numbers. The cavity number remains one of the most peculiar properties of the free volume whose physical meaning remains obscure. The cavity number or the number of free volume holes is obtained experimentally from the PALS measurements when it is supposed that it is related to the intensity of the PALS signal, i.e., probability of o-Ps formation.

Figure $7 \mathrm{a}$ shows the cavity number as a function of probe radius for three different temperatures. As the radius of the probe increases, the free volume structure breaks into separate cavities that represent pockets of free volume created by surrounding molecular bodies, i.e., water molecules or polymer segments. Figure $7 \mathrm{a}$ shows that with increasing temperature the distribution of cavity numbers spreads and the position of the cavity number maxima shifts toward higher probe radii. The spreading of the distribution arises from opening the molecular structure, while more of the molecular surface becomes accessible to the probe with decreasing density. ${ }^{31}$

The distributions are calculated starting from the probe radius with $0.5 \AA$ where the number density of cavities already reaches a value of 0.001 cavity per $\AA^{3}$. However, as it was shown earlier the cavity number density starts from $1 / V_{\text {tot }}$ since a point probe with zero radius can sample the free volume spaces in molecular structure so effectively that the free volume is contained in one single cavity percolating the structure. $^{31}$ The percolating cavity creates a complex structure of free volume pockets interconnected by bottlenecks. To analyze the free volume in terms of cavities, the free volume pockets need to be determined in the structure. In the work by Jansen et al., ${ }^{79}$ the authors suggested that in more rigid polymers with large side groups that create large free volumes the free volume structure could be obtained by using probes with increased radii such as 1.5 up to $2.5 \AA$ which represent smaller molecules or an upper limit of the orthopositronium radius. Using arbitrarily large probes to determine cavity structure would encounter two major problems: (i) if an increased probe radius is used, the free volume certainly breaks into separate cavities, but the free volume amounts determined by larger probe radii dramatically drop (see Figure 3), making the obtained quantity impossible to be compared with definitions in existing free volume theories; (ii) using arbitrarily large probes for different systems would make a systematic comparison of the calculated free volume in different systems impossible.

To address the first problem, the two-parameter model was developed and used to investigate free volumes in pure PVME, $^{31}$ and it was described in detail in a separate paper. ${ }^{52}$ In this model, the free volume is at first calculated by employing a small probe of e.g. $R_{\mathrm{p} 1}=0.5 \AA$. Next, the free volume pockets representing cavities are determined by probe with a large radius, $R_{\mathrm{p} 2}$, while the free volume determined by the smaller probe is associated with a particular pocket based on the criteria of distance. This approach preserves the amounts of the free volume present in the molecular structure, but it opens a question of the larger probe radius used to determine the actual cavities. As mentioned earlier, cavity number is result of geometry of intermolecular space, and its physical meaning has to be associated with a particular physical property. In the PALS experiment, the number of cavities is supposed to be related to the intensity of o-Ps annihilation signal $I_{3}$ that represents the probability of o-Ps formation. ${ }^{93}$ Experimentally, the dependence of intensity $I_{3}$ on temperature shows a complex behavior that varies from one polymeric system to another, while the measured intensity can rise or decrease with temperature. So far, the origin of such the behavior has not been satisfactorily explained. Thus, to ease the treatment of experimental data, the cavity number in experimental works was often approximated to a constant. ${ }^{93-95}$

We would like to stress that a constant number of cavities cannot be geometrically justified, and it is proprietary only to the free volume definitions based on Voronoi tessellation and its complementary Delaunay tessellation of space. In the tessellation approach, the volume is dissected into polyhedrons that represent volume associated with a particular atom, hence implying a constant number of polyhedrons equal to number of atoms, while the tessellation does not provide information about changes in a solvent accessible surface. Rather than using the cavity number constant, we investigated the number of cavities as a function of temperature and probe radii used (Figure $7 \mathrm{~b}$ ). The temperature dependences show a complex behavior, where for small probes with radii below the cavity number maximum (shown in Figure $7 \mathrm{a}$ ) the number of cavities with temperature drops, as the cavities tend to aggregate as the structure opens. Similar behavior of the intensity $I_{3}$ was observed experimentally in glycerol and discussed also with computed cavity numbers. ${ }^{69}$ For large probes, the cavity number and hence the number of the free volume pockets increase with temperature. This is because the number of holes able to contain such large probes increases as the structure expands with increasing temperature. Similar characteristic behavior is experimentally measured for $I_{3}$ in pure PVME. ${ }^{96}$ By comparing temperature dependence of cavity number with the temperature dependence of the intensity $I_{3}$, we chose the value of the second parameter to be the radius of slightly above the cavity number maximum, $R_{\mathrm{p} 2}=0.9 \AA^{31}$

By fixing the cavity number to be around its maximum in the region deep below $T_{g}$, a percolation of the cavity volumes would be observed with increasing temperature, with the major onset of percolation around the region of the glass transition. Percolation of the free volume is an important part of the free volume theory introduced by Cohen and Grest. ${ }^{97-99}$ The percolation model assumes the existence of liquidlike and solidlike molecules, while exchange of the free volume between the molecules could happen only between the liquidlike ones. Then, the glass transition would be associated with percolation of the liquidlike molecules in the structure. Voloshin et al. put the percolation theory to the test by computing volumes of Voronoi polyhedrons in a model of Lennard-Jones liquid, but they concluded that the liquid-to-amorphous solidlike transition is not associated with percolation through regions with a large free volume since the percolation thresholds they obtained in amorphous solid and liquid did not practically differ. $^{100}$

We believe the conclusion of Voloshin et al. was biased by the method used to compute free volume around their beads (molecules). Voronoi tessellation arbitrarily keeps the number of cells, i.e., cavities, equal to the number of molecules and 
does not allow calculation of the cavity volumes based on the probe accessible surface. As such, the Voronoi polyhedrons provide information about the free volume in the first coordinating shell around atoms. As we have shown, the cavity-cavity pair distribution function providing information about free volumes in coordination shells is complementary to the atomic radial distribution function; therefore, it is not a good quantity to distinguish between glassy and liquid structures. $^{84}$ The character of the pair distribution function in the work of Voloshin et al. is evident in their attempt to address the $z$-percolation problem, where $z$ denotes number of liquidlike neighbors that each molecule needs to have before it is accounted to the percolation cluster. In the Voronoi distribution the cases of $z=0,1$, and 2 represent the same case.

In Figure 8, we show the picture on the free volume percolation by computing integral distributions of the total free

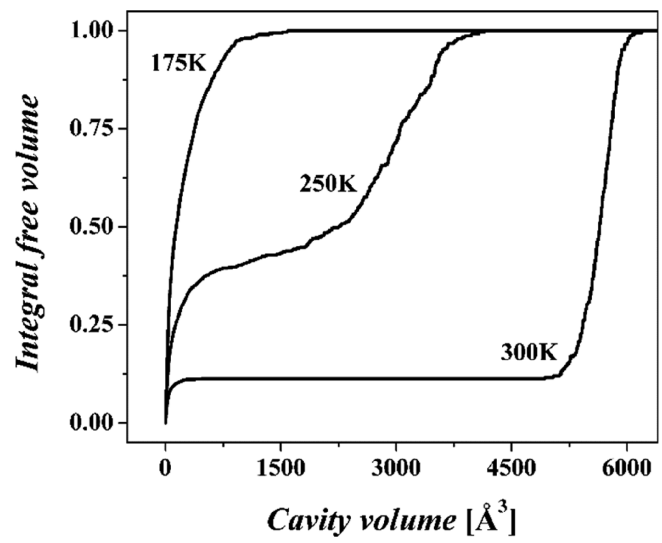

Figure 8. Percolation of the free volume. The integral free volume is given as a function of the cavity volume and of temperature as indicated.

volume as a function of the cavity volume. The absolute values of the free volume are normalized to unity. The graph indicates the percolation by change of the trend of the distribution at simulated $T_{\mathrm{g}}^{\mathrm{MD}}=250 \mathrm{~K}$. The percolation threshold, i.e., the amount of the free volume, would correspond to the value computed for a probe with radius of $0.5 \AA$ (Figure 2) and the fraction of the free volume at $T_{\mathrm{g}} \sim 11 \%$. The critical size of the percolating cluster that was found for a free volume cavity observed to be interconnected through the periodic boundaries of the simulation box is around $3500 \AA^{3}$. At temperatures well above $T_{\mathrm{g}}$ basically all the free volume $(90 \%)$ is interconnected in percolating cavity of infinite size. The remaining $10 \%$ of the free volume cavities not involved in the percolated cavity correspond to the interstitial volume. Because of fractal character of the cavities, the coordination of molecules, i.e., water molecules and polymer segments, with cavities follows a complex dependence as observed for the number of cavities shown in Figure $7 a, b{ }^{31}$

PALS Free Volume. Experimental measurement of the free volume by using positronium annihilation for a polymer in aqueous solution would be complicated even if possible due to the known effect of water causing inhibition of positronium formation. ${ }^{38}$ In the positronium annihilation study of polyimides containing water, Dlubek et al. suggested that oPs forms only in the polymer cavities that do not contain water molecules. ${ }^{101}$ In Figure 3, we showed distributions of the free volume in three different environments. The first prediction for the free volume in possible future PALS measurements, based on our computational model and Dlubek's assumption, is that the free volume fraction would be $\sim 10$ times lower than usually measured by means of the PALS measurements, since the fraction of the free volume contained solely in the polymer phase was found to be only a tenth of the total free volume. The peak of the size distribution of free volume cavities formed solely by polymer is centered at position $33.9 \pm 0.1 \AA^{3}\left(R^{2}=\right.$ $0.9806)$. The median of the corresponding distribution represents the mean free hole volume $40.1 \pm 0.5 \AA^{3}$. By use of the standard quantum mechanical model of o-Ps in a spherical hole, ${ }^{102}$ the o-Ps lifetime corresponding to the mean cavity volume in polymer phase is calculated $\tau_{3}=1.31 \mathrm{~ns}$. The median of the cavity volume in polymeric environment is considerably lower than an average volume computed for the whole structure, $102.6 \pm 1.0 \AA^{3}$. This average cavity volume correlates with the o-Ps lifetime of $\tau_{3}=2.03 \mathrm{~ns}$. The calculated lifetime has the same value as obtained for the pure PVME. ${ }^{31}$ As discussed earlier, despite a larger specific volume of the swollen wet PVME, the presence of small water molecules results in creating effectively smaller cavities, and as result the mean o-Ps lifetime in PVME solution is the same as in the pure PVME. A similar effect of water was observed on glycerol solutions, where the o-Ps lifetime showed no change with varying content of water. ${ }^{92}$

\section{CONCLUSIONS}

By means of molecular dynamics simulations and simulated cooling, we have prepared molecular structures of poly(vinyl methyl ether) aqueous solution with water content of $c_{\mathrm{w}}=30 \%$ in a wide temperature range $400 \mathrm{~K}$ down to $175 \mathrm{~K}$. In the simulated structures, we have investigated the free volume microstructure in terms of various free volume measures, such as free volume fractions, free volume distributions, cavity numbers, and cavity environment. The free volume obtained computationally by geometrical analyses of the intermolecular space is a direct method for obtaining the free volume. The free volume calculations are still computationally demanding, but the development of new computational methods able to obtain various properties of the free volume such as its environment, dynamics, distributions, etc., will make it possible to revisit conclusions by various free volume definitions after more data are collected.

In the current work we show that the behavior of the excess free volume, calculated for a probe with a radius $0.5 \AA$, agrees with the predictions by Simha-Boyer theory in terms of the free volume at $\left(\alpha_{\mathrm{L}}-\alpha_{\mathrm{G}}\right) T_{\mathrm{g}} \sim 11 \%$ and the coefficient of thermal expansion $\alpha_{\mathrm{L}}^{f}=\alpha_{\mathrm{L}}-\alpha_{\mathrm{G}}$. A good agreement would be obtained also with the recent LCL model, predicting the value of fractional free volume at $T_{\mathrm{g}}^{\mathrm{MD}}$ at $7.3 \%$, if a probe with slightly increased radius is employed, $R_{\mathrm{p}}=0.6 \AA$. Moreover, the obtained free volume data were found consistent with the concentric shell model and data from the PALS measurements, which confirmed that the computed free volume data are physically relevant and reasonable for further discussion of the free volume distributions around specific sites around molecules of plasticizer.

The calculated free volume distributions showed that at the concentrations $c_{\mathrm{w}}=30 \%$ of water the water is perfectly distributed around the polymer chains, with $90 \%$ of the free volume cavities formed by both water and polymeric molecules. The remaining $10 \%$ of the cavities is formed by 
polymer segments or by water molecules and corresponded in a greater part to interstitial cavities. This picture corresponds to experimental findings where at lower concentrations of water the water molecules are complexed to the polymer chain, rather than forming a continuous phase.

An interesting observation was made for the free volume around polymer ends, which are expected to be associated with larger free volumes. However, as simulations have shown, the increase is only by a subtle $7 \%$. This is probably due to the presence of the water molecules, which have been shown to take control over polymer chain dynamics by experimental dynamics studies (QENS) or as suggested by combined Raman spectroscopy, dielectric spectroscopy, and calorimetry experimental work.

Furthermore, multiplicity of hydrogen bonds is in excellent agreement with the free volume-mobility relationship encoded in the free volume theory. The computed free volume distributions have shown that around sites with increased hydrogen-bonding formation, and hence smaller mobility, lower amounts of the free volume are directly determined.

Besides the implications for the free volume theory, the computed average cavity volumes, being around $102 \AA^{3}$ in average and $40 \AA^{3}$ solely in polymer, provide information about pore sizes available to diffusants in the hydrogel formed by PVME and water. The pore size is an important property for biomedical applications of hydrogel such as membranes or drug carriers. The computed mean cavity volumes are also interesting for the experimental measurements by means of positronium annihilation spectroscopy, while the aim here is creating a complementary rather than supplementary tool for direct measurements of the free volume.

Toward the ongoing development of hybrid methods employing combined tessellation-voxelization of space by Manak et al., ${ }^{43}$ it would be challenging to include an aspect of time autocorrelation functions for determination of the excess free volume cavities, such as can be obtained by excessive probing of time-correlated structures, presented earlier. ${ }^{76}$

\section{AUTHOR INFORMATION}

\section{Corresponding Author}

Dušan Račko - Polymer Institute, Slovak Academy of Sciences, 84145 Bratislava, Slovak Republic; 이잉.org/0000-00029203-9652; Email: dusan.racko@savba.sk

\section{Authors}

Sara Capponi - Department of Industrial and Applied Genomics, IBM AI and Cognitive Software Organization, IBM Almaden Research Center, San Jose, California 95120-6099, United States; NSF Center for Cellular Construction, University of California, San Francisco, San Francisco, California 94115, United States; 10 orcid.org/0000-0001-8117-7526

Fernando Alvarez - Centro de Fisica de Materiales (CFM) (CSIC-UPV/EHU) - Materials Physics Center (MPC), 20018 San Sebastián, Spain; Departamento de Fisica de Materiales, UPV/EHU, 20080 San Sebastián, Spain; ㅈorcid.org/00000002-7038-0315

Complete contact information is available at: https://pubs.acs.org/10.1021/acs.macromol.0c00472

\section{Notes}

The authors declare no competing financial interest.

\section{ACKNOWLEDGMENTS}

D.R. acknowledges support by the Grant Agency of the Ministry of Education, Science, Research and Sport of the Slovak Republic, Grant VEGA 2/0102/20, and support is also acknowledged from the Slovak Research and Development Agency, Grants SRDA 160369 and SRDA 15 0323. F.A. gratefully acknowledges the financial support of the Basque Government, code IT-1175-19, and the Ministerio de Economía y Competitividad, code PGC2018-094548-B-100 (MINECO/FEDER, UE).

\section{REFERENCES}

(1) Kricheldorf, H. R; Nuyken, O.; Swift, G. Handbook of Polymer Synthesis, 2nd ed.; CRC Press Inc.: Boca Raton, FL, 2004.

(2) Schildknecht, C. E. Vinyl and Related Polymers; Wiley: New York, 1952; p 593.

(3) Schildknecht, C. E.; Zoss, A. O.; McKinley, C. Vinyl Alkyl Ethers. Ind. Eng. Chem. 1947, 39, 180. (1947).

(4) Gil, E. S.; Hudson, S. M. Stimuli-reponsive polymers and their bioconjugates. Prog. Polym. Sci. 2004, 29, 1173-1222.

(5) Janik, I.; Ulanski, P.; Rosiak, J. M. Pulse radiolysis of poly (vinyl methyl ether) in aqueous solution. Formation and structure of primary radicals. Nucl. Instrum. Methods Phys. Res., Sect. B 1999, 151, $318-323$.

(6) Arndt, K.-F.; Schmidt, T.; Menge, H. Poly (vinyl methyl ether) hydrogel formed by high energy irradiation. Macromol. Symp. 2001, $164,313-22$.

(7) Frieberg, B.; Kim, J.; Narayanan, S.; Green, P. F. Surface dynamics of miscible polymer blend nanocomposites. ACS Nano 2014, 8, 607-613.

(8) Molyneux, P. Water-Soluble Synthetic Polymers: Properties and Behavior; CRC Press Inc.: Boca Raton, FL, 1983; Vol. I.

(9) Capponi, S.; Arbe, A.; Cerveny, S.; Busselez, R.; Frick, B.; Embs, J. P.; Colmenero, J. Quasielastic neutron scattering study of hydrogen motions in an aqueous poly (vinyl methyl ether) solution. J. Chem. Phys. 2011, 134, 204906.

(10) Cerveny, S.; Schwartz, G. A.; Bergman, R.; Swenson. Glass transition and relaxation processes in supercooled water. Phys. Rev. Lett. 2004, 93, 245702.

(11) Cerveny, S.; Colmenero, J.; Alegria, A. Dielectric Investigation of the Low-Temperature Water Dynamics in the Poly(vinyl methyl ether) $/ \mathrm{H}_{2} \mathrm{O}$ System. Macromolecules 2005, 38, 7056-7063.

(12) Higuchi, A.; Komiyama, J.; Iijima. The states of water in gel cellophane membranes. Polym. Bull. (Heidelberg, Ger.) 1984, 11, 203208.

(13) de Dood, M. J. A.; Kalkman, J.; Strohhofer, C.; Michielsen, J.; van der Elsken, J. Hidden transition in the "unfreezable water" region of the PVP- water system. J. Phys. Chem. B 2003, 107, 5906-5913. (14) Meeussen, F.; Bauwens, Y.; Moerkerke, R.; Nies, E.; Berghmans, H. Molecular complex formation in the system poly (vinyl methyl ether)/water. Polymer 2000, 41, 3737-3743.

(15) Zeng, X.; Yang, X. A study of interaction of water and model compound of poly (vinyl methyl ether). J. Phys. Chem. B 2004, 108, 17384-17392.

(16) Tamai, Y.; Tanaka, H.; Nakanishi, K. Molecular dynamics study of polymer-water interaction in hydrogels. 2. Hydrogen-bond dynamics. Macromolecules 1996, 29, 6761-6769.

(17) Tamai, Y.; Tanaka, H.; Nakanishi, K. Molecular dynamics study of polymer-water interaction in hydrogels. 1. Hydrogen-bond structure. Macromolecules 1996, 29, 6750-6760.

(18) Pastorczak, M.; Kozanecki, M.; Ulanski, J. Water-Polymer interactions in PVME hydrogels-Raman spectroscopy studies. Polymer 2009, 50, 4535-4542.

(19) Roland, C. M. Relaxation phenomena in vitrifying polymers and molecular liquids. Macromolecules 2010, 43, 7875-7890.

(20) Cangialosi, D. Dynamics and thermodynamics of polymer glasses. J. Phys.: Condens. Matter 2014, 26, 153101. 
(21) Debenedetti, P. G Metastable Liquids; Princeton University Press: Princeton, NJ, 1996.

(22) Goldstein, M. Viscous Liquids and the Glass Transition. IV. Thermodynamic Equations and the Transition. J. Phys. Chem. 1973, 77, 667-673.

(23) White, R. P.; Lipson, J. E. G. Polymer free volume and its connection to the glass transition. Macromolecules 2016, 49, 39874007.

(24) Jean, Y. C.; Mallon, P. E.; Schrader, D. M. Principles and Applications of Positron \& Positronium Chemistry; World Scientific Publishing: Singapore, 2002; p 255.

(25) Tao, S. J. Positronium annihilation in molecular substances. J. Chem. Phys. 1972, 56, 5499.

(26) Eldrup, M.; Lightbody, D.; Sherwood, J. N. The temperature dependence of positron lifetimes in solid pivalic acid. Chem. Phys. 1981, 63, 51 .

(27) Nakanishi, H.; Wang, S. J.; Jean, Y. C. In Positron Annihilation Studies of Fluids; Sharma, S. C., Ed.; World Science: Singapore, 1988. (28) Jean, Y. C.; Shi, H. J. Positronium lifetime in an ellipsoidal freevolume hole of polymers. J. Non-Cryst. Solids 1994, 172-174, 806814.

(29) Jasińska, B.; Koziol, A. E.; Goworek, T. Ortho-positronium lifetimes in nonspherical voids. J. Radioanal. Nucl. Chem. 1996, 210, 617-623.

(30) Olson, B. G.; Prodpran, T.; Jamieson, A. M.; Nazarenko, S. Positron annihilation in syndiotactic polystyrene containing $\alpha$ and $\beta$ crystalline forms. Polymer 2002, 43, 6775-6784.

(31) Racko, D.; Capponi, S.; Alvarez, F.; Colmenero, J. The free volume of poly (vinyl methylether) as computed in a wide temperature range and at length scales up to the nanoregion. J. Chem. Phys. 2011, 134, 044512.

(32) Consolati, G.; Panzarasa, G.; Quasso, F. Morphology of free volume holes in an amorphous polyether-polyester polyurethane of biomedical interest. Polym. Test. 2018, 68, 208-212.

(33) McCullagh, C. M.; Yu, Z.; Jamieson, A. M.; Blackwell, J.; McGervey, J. D. Positron Annihilation Lifetime Measurements of Free Volume in Wholly Aromatic Copolyesters and Blends. Macromolecules 1995, 28, 6100-6107.

(34) Dlubek, G.; Saarinen, K.; Fretwell, H. M. The Temperature Dependence of the Local Free Volume in Polyethylene and Polytetrafluoroethylene: A Positron Lifetime Study. J. Polym. Sci., Part B: Polym. Phys. 1998, 36, 1513-1528.

(35) Bandžuch, P.; Krištiak, J.; Šauša, O.; Zrubcová, J. Direct computation of the free volume fraction in amorphous polymers from positron lifetime measurements. Phys. Rev. B: Condens. Matter Mater. Phys. 2000, 61, 8784.

(36) Consolati, J. On the Thermal Expansion of Nanohole Free Volume in Perfluoropolyethers. J. Phys. Chem. B 2005, 109, 1009610099.

(37) Shantarovich, V. P.; Suzuki, T.; He, Ch.; Gustov, V. W. Inhibition of positronium formation by polar groups in polymersrelation with TSL experiments. Radiat. Phys. Chem. 2003, 67, 15-23.

(38) Mogensen, O. E. Positron Annihilation in Chemistry; SpringerVerlag: Heidelberg, 1995.

(39) Rycroft, C. H.; Grest, G. S.; Landry, J. W.; Bazant, M. Z. Analysis of granular flow in a pebble-bed nuclear reactor. Phys. Rev. E Stat. Nonlinear Soft Matter Phys. 2006, 74, 021306.

(40) Su, P.; Drysdale, R. L. S. A comparison of sequential Delaunay triangulation algorithms. Comput. Geom. 1997, 7, 361-385.

(41) Rigby, D.; Roe, R. J. Molecular dynamics simulation of polymer liquid and glass. 4. Free-volume distribution. Macromolecules 1990, 23, 5312.

(42) Connolly, M. L. Analytical molecular surface calculation. J. Appl. Crystallogr. 1983, 16, 548-558.

(43) Manak, M.; Jirkovsky, L.; Kolingerova, I. Interactive analysis of Connolly surfaces for various probes. Comput. Graph. Forum 2017, 36,160 .
(44) Putta, S.; Nemat-Nasser, S. Molecularly-based numerical evaluation of free volume in amorphous polymers. Mater. Sci. Eng., A 2001, 317, 70-76.

(45) Adcock, S. A.; McCammon, J. A. Molecular dynamics: survey of methods for simulating the activity of proteins. Chem. Rev. 2006, 106, $1589-1615$.

(46) Bunte, S. W.; Sun, H. Molecular modeling of energetic materials: the parameterization and validation of nitrate esters in the COMPASS force field. J. Phys. Chem. B 2000, 104, 2477.

(47) Sun, H. COMPASS: an ab initio force-field optimized for condensed-phase applications overview with details on alkane and benzene compounds. J. Phys. Chem. B 1998, 102, 7338.

(48) Yang, J.; Ren, Y.; Tian, A.-m.; Sun, H. COMPASS Force Field for 14 Inorganic Molecules, $\mathrm{He}, \mathrm{Ne}, \mathrm{Ar}, \mathrm{Kr}, \mathrm{Xe}, \mathrm{H}_{2}, \mathrm{O}_{2}, \mathrm{~N}_{2}, \mathrm{NO}, \mathrm{CO}$, $\mathrm{CO}_{2}, \mathrm{NO}_{2}, \mathrm{CS}_{2}$, and $\mathrm{SO}_{2}$, in Liquid Phases. J. Phys. Chem. B 2000, 104, 4951.

(49) Theodorou, D. N.; Suter, U. W. Detailed molecular structure of a vinyl polymer glass. Macromolecules 1985, 18, 1467.

(50) Frenkel, D.; Smit, B. Understanding Molecular Simulation: From Algorithms to Applications; Academic Press: 2002.

(51) Colmenero, J.; Alvarez, F.; Arbe, A. Self-motion and the $\alpha$ relaxation in a simulated glass-forming polymer: Crossover from Gaussian to non-Gaussian dynamic behaviour. Phys. Rev. E: Stat. Phys., Plasmas, Fluids, Relat. Interdiscip. Top. 2002, 65, 041804.

(52) Racko, D. A Computational Model for Nano Scale Cavities in the Atomic Structure of Polymer Melt and Comparisons to PALS. Mater. Sci. Forum 2010, 666, 15-20.

(53) Hempel, E.; Hempel, G.; Hensel, A.; Schick, C.; Donth, E. Characteristic Length of Dynamic Glass Transition near $\mathrm{Tg}$ for a Wide Assortment of Glass-Forming Substances. J. Phys. Chem. B 2000, 104, 2460-2466.

(54) Alba-Simionesco, C.; Cailliaux, A.; Alegria, A.; Tarjus, G. Scaling out the density dependence of the $\alpha$ relaxation in glassforming polymers. Europhys. Lett. 2004, 68, 58-64.

(55) Loozen, E.; Van Durme, K.; Nies, E.; Van Mele, B.; Berghmans, $\mathrm{H}$. The anomalous melting behavior of water in aqueous PVME solutions. Polymer 2006, 47, 7034-7042.

(56) Baschnagel, J; Binder, K; Wittmann, H.-P. The influence of the cooling rate on the glass transition and the glassy state in threedimensional dense polymer melts: a Monte Carlo study. J. Phys.: Condens. Matter 1993, 5, 1597-1618.

(57) Schawe, J. E. K. Measurement of the thermal glass transition of polystyrene in a cooling rate range of more than six decades. Thermochim. Acta 2015, 603, 128-134.

(58) Schäfer-Soenen, H.; Moerkerke, R.; Berghmans, H.; Koningsveld, R.; Dušek, K.; Šolc, K. Zero and Off-Zero Critical Concentrations in Systems Containing Polydisperse Polymers with Very High Molar Masses. 2. The System Water-Poly(vinyl methyl ether). Macromolecules 1997, 30, 410-416.

(59) Maeda, H. Interaction of water with poly (vinyl methyl ether) in aqueous solution. J. Polym. Sci., Part B: Polym. Phys. 1994, 32, 9197.

(60) Meeussen, F.; Bauwens, Y.; Moerkerke, R.; Nies, E.; Berghmans, H. Molecular complex formation in the system poly (vinyl methyl ether)/water. Polymer 2000, 41, 3737-3743.

(61) Angell, C. A. Liquid fragility and the glass transition in water and aqueous solutions. Chem. Rev. 2002, 102, 2627-2650.

(62) Zhou, Y.; Milner, S. T. Average and Local Tg Shifts of Plasticized PVC from Simulations. Macromolecules 2018, 51, 38653873.

(63) Holten, V.; Anisimov, M. A. Entropy-driven liquid-liquid separation in supercooled water. Sci. Rep. 2012, 2, 713-719.

(64) Fox, T. G.; Flory, P. J. Second-order transition temperatures and related properties of polystyrene. I. Influence of molecular weight. J. Appl. Phys. 1950, 21, 581-591.

(65) Steinhaus, H. Mathematical Snapshots, 3rd ed.; Dover: New York, 1999; pp 202-203. 
(66) Doolittle, A. K. Studies in Newtonian flow. I. The dependence of the viscosity of liquids on temperature. J. Appl. Phys. 1951, 22, 1031.

(67) Williams, M. L.; Landel, R. F.; Ferry, J. D. The temperature dependence of relaxation mechanisms in amorphous polymers and other glass-forming liquids. J. Am. Chem. Soc. 1955, 77, 3701.

(68) Simha, R.; Boyer, R. F. On a general relation involving the glass temperature and coefficients of expansion of polymers. J. Chem. Phys. 1962, 37, 1003

(69) Racko, D.; Chelli, R.; Cardini, G.; Bartos, J.; Califano, S. Insights into positron annihilation lifetime spectroscopy by molecular dynamics simulations. Eur. Phys. J. D 2005, 32, 289-297.

(70) Martin, J. M.; Rogers, S. S.; Mandelkern, L. Volumetemperature relations of amorphous polymers over on extended temperature range. J. Polym. Sci. 1956, 20, 579.

(71) White, R. P.; Lipson, J. E. G. Free volume, cohesive energy density, and internal pressure as predictors of polymer miscibility. Macromolecules 2014, 47, 3959-3968.

(72) White, R. P.; Lipson, J. E. G. Free volume in the melt and how it correlates with experimental glass transition temperatures: results for a large set of polymers. ACS Macro Lett. 2015, 4, 588-592.

(73) White, R. P.; Lipson, J. E. G.; Higgins, J. S. How Pure Components Control Polymer Blend Miscibility. Macromolecules 2012, 45, 8861-8871.

(74) White, R. P.; Lipson, J. E. G.; Higgins, J. S. New Correlations in Polymer Blend Miscibility. Macromolecules 2012, 45, 1076-1084.

(75) White, R. P.; Lipson, J. E. G. Connecting Theory and Experiment To Understand Miscibility in Polymer and Small Molecule Mixtures. J. Chem. Eng. Data 2014, 59, 3289-3300.

(76) Racko, D.; Krištiak, J. Free Volume Dynamics. Mater. Sci. Forum 2012, 733, 33-37.

(77) Arizzi, S.; Mott, P. H.; Suter, U. W. Space available to small diffusants in polymeric glasses: analysis of unoccupied space and its connectivity. J. Polym. Sci., Part B: Polym. Phys. 1992, 30, 415.

(78) Vrentas, J. S.; Duda, J. L. Molecular diffusion in polymer solutions. AIChE J. 1979, 25, 1-24.

(79) Jansen, J. C.; Macchione, M.; Tocci, E.; De Lorenzo, L.; Yampolskii, Y. P.; Sanfirova, O.; Shantarovich, V. P.; Heuchel, M.; Hofmann, D.; Drioli, E. Comparative study of different probing techniques for the analysis of the free volume distribution in amorphous glassy perfluoropolymers. Macromolecules 2009, 42, 7589.

(80) Švajdlenková, H.; Iskrová, M.; Šauša, O.; Dlubek, G.; Krištiak, J.; Bartoš, J. The Spin Probe Dynamics and the Free Volume in a Series of Amorphous Polymer Glass-Formers. Macromol. Symp. 2011, 305, 108-115.

(81) Švajdlenková, H.; Šauša, O.; Iskrová-Miklošovičová, M.; Majerník, V.; Krištiak, J.; Bartoš, J. On the relationships between guest molecular dynamics and free volume in a series of small molecular and polymer glass-formers. Chem. Phys. Lett. 2012, 539$540,39-44$

(82) Rikvold, P. A.; Stell, G. D-dimensional interpenetrable-sphere models of random two-phase media: microstructure and an application to chromatography. J. Colloid Interface Sci. 1985, 108, 158.

(83) Misra, S.; Mattice, W. Atomistic models of amorphous polybutadienes. 3. Static free volume. Macromolecules 1993, 26, 7274.

(84) Racko, D.; Capponi, S.; Alvarez, F.; Bartos, J.; Colmenero, J. The free-volume structure of a polymer melt, poly (vinyl methylether) from molecular dynamics simulations and cavity analysis. J. Chem. Phys. 2009, 131, 064903.

(85) Heuchel, M.; Hofmann, D.; Pullumbi, P. Molecular modeling of small-molecule permeation in polyimides and its correlation to freevolume distributions. Macromolecules 2004, 37, 201.

(86) Schmidtke, E.; Gunther-Schade, K.; Hofmann, D.; Faupel, F. The distribution of the unoccupied volume in glassy polymers. J. Mol. Graphics Modell. 2004, 22, 309.

(87) Harmandaris, V. A.; Doxastakis, M.; Mavrantzas, V. G.; Theodorou, D. N. Detailed molecular dynamics simulation of the selfdiffusion of n-alkane and cis-1,4 polyisoprene oligomer melts. J. Chem. Phys. 2002, 116, 436.
(88) Bondi, A. van der Waals volumes and radii. J. Phys. Chem. 1964, $68,441$.

(89) Fox, T. G. Influence of diluent and of copolymer composition on the glass temperature of a polymer system. Bull. Am. Phys. Soc. 1956, 1, 123.

(90) Pastorczak, M.; Dominguez-Espinosa, G.; Okrasa, L.; Pyda, M.; Kozanecki, M.; Kadlubowski, S.; Rosiak, J. M.; Ulanski, J. Poly (vinyl methyl ether) hydrogels at temperatures below the freezing point of water-molecular interactions and states of water. Colloid Polym. Sci. 2014, 292, 1775-1784.

(91) Townrow, S.; Roussenova, M.; Giardiello, M.-I.; Alam, A.; Ubbink, J. Specific volume- hole volume correlations in amorphous carbohydrates: Effect of temperature, molecular weight, and water content. J. Phys. Chem. B 2010, 114, 1568-1578.

(92) Roussenova, M.; Murith, M.; Alam, A.; Ubbink, J. Plasticization, antiplasticization, and molecular packing in amorphous carbohydrate-glycerol matrices. Biomacromolecules 2010, 11, 32373247.

(93) Wang, Y. Y.; Nakanishi, H.; Jean, Y. C.; Sandreczki, T. C. Positron annihilation in amine-cured epoxy polymers-pressure dependence. J. Polym. Sci., Part B: Polym. Phys. 1990, 28, 1431-1441.

(94) Krištiak, J.; Bartoš, J.; Krištiaková, K.; Sauša, O.; Bandžuch, P. Free-volume microstructure of amorphous polycarbonate at low temperatures determined by positron-annihilation-lifetime spectroscopy. Phys. Rev. B: Condens. Matter Mater. Phys. 1994, 49, 66016608.

(95) Consolati, G.; Levi, M.; Messa, L.; Tieghi, G. Free volumes and occupied volumes in oligomeric polypropylenglycols. Europhys. Lett. 2001, 53, 497.

(96) Račko, D. On the Relationship of the Relative Intensity $I_{3}$ and a Cavity Number as Obtained from Computer Simulations. Mater. Sci. Forum 2012, 733, 183-189.

(97) Cohen, M. H.; Grest, G. S. Liquid-glass transition, a freevolume approach. Phys. Rev. B: Condens. Matter Mater. Phys. 1979, 20, 1077.

(98) Cohen, M. H.; Grest, G. S. Dispersion of relaxation rates in dense liquids and glasses. Phys. Rev. B: Condens. Matter Mater. Phys. 1981, 24, 4091-4094.

(99) Grest, G. S.; Cohen, M. H. Liquids, glasses, and the glass transition: A free-volume approach. Adv. Chem. Phys. 2007, 48, 455.

(100) Voloshin, V. P.; Naberukhin, Y. I.; Medvedev, N. N.; Jhon, M. $S$. Investigation of free volume percolation under the liquid-glass phase transition. J. Chem. Phys. 1995, 102, 4981.

(101) Dlubek, G.; Buchhold, R.; Huebner, Ch.; Nakladal, A. Water in local free volumes of polyimides: A positron lifetime study. Macromolecules 1999, 32, 2348-2355.

(102) Nakanishi, H.; Wang, S. J.; Jean, Y. C. In Positron Annihilation Studies of Fluids; Sharma, S. S., Ed.; World Scientific: Singapore, 1988; p 292. 